\title{
Cardiovascular and Renal Benefits of SGLT2 Inhibitors: A Narrative
}

\section{Review}

\author{
Soghra Rabizadeh ${ }^{1}$, Manouchehr Nakhjavani ${ }^{1}$ and Alireza Esteghamati ${ }^{1,{ }^{*}}$ \\ ${ }^{1}$ Endocrinology and Metabolism Research Center (EMRC), Vali-Asr Hospital, Tehran University of Medical Sciences, Tehran, Iran \\ "Corresponding author: Endocrinology and Metabolism Research Center (EMRC), Vali-Asr Hospital, Tehran University of Medical Sciences, Tehran, Iran. Email: \\ esteghamati@tums.ac.ir
}

Received 2018 September 16; Revised 2019 February 27; Accepted 2019 March 03.

\begin{abstract}
Context: Most recently developed anti-hyperglycemic drugs have offered cardiovascular and renal benefits. In this narrative review, we discuss the cardiovascular and renal benefits of novel antidiabetic drugs, sodium glucose cotransporter type 2 (SGLT2) inhibitors, in type 2 diabetes.

Evidence Acquisition: The literature published in PubMed, Scopus, Web of Science, Google Scholar, and Cochrane library were reviewed up to January 2019. The keywords including SGLT2 inhibitor, type 2 diabetes, cardiovascular effect, and renal effect were used in different combinations.

Results: Cardiovascular disease represents a large health burden in patients with diabetes. The prevention of cardiovascular events is a major concern in the treatment of patients with diabetes. Diabetes is also associated with an increased risk of adverse renal events and diabetic nephropathy is the leading cause of end-stage renal disease worldwide. SGLT2 inhibitors as new glucoselowering agents act by inhibiting glucose reabsorption in the proximal tubule of the kidney, which is independent of insulin secretion. We reviewed the cardiovascular effects of these drugs including effects on triple MACE (major adverse cardiovascular events), myocardial infarction, heart failure, cardiovascular and all-cause mortality, and stroke, as well as renal effects including albuminuria, serum creatinine, the rate of renal replacement therapy, and renal function over time, along with the mechanisms of these effects.

Conclusions: Given the suboptimal glycemic and cardiovascular risk control in type 2 diabetes, novel therapies such as SGLT2 inhibitors seem to have an important clinical advantage to improve glycemic control and cardiovascular and renal outcomes.
\end{abstract}

Keywords: Diabetes Mellitus, Sodium-Glucose Cotransporter 2 Inhibitors, Cardiovascular Disease, Chronic Kidney Disease

\section{Context}

Cardiovascular disease imposes a large health burden in patients with diabetes $(1,2)$. Type 2 diabetes is associated with about a twofold increase in the risk of a wide range of cardiovascular diseases (3). Mortality risk after the first incidence of myocardial infarction is higher in patients with diabetes than in those without diabetes (4). The prevention of cardiovascular events, therefore, is a major concern in the treatment of patients with diabetes.

Recently, one study in a university-affiliated clinic in Iran investigated the effect of antidiabetic medications, including metformin, glibenclamide plus metformin, insulin alone, and insulin plus metformin, on pulse pressure and blood pressure, showing no significant difference between any of these anti-hyperglycemic drugs (5). Unfortunately, there are no nationally representative data on the mortality of diabetes patients undergoing treatment with different oral antidiabetic drugs. We studied this issue in a relatively large sample in our diabetes center, which revealed that treatment with glyburide was correlated with all-cause mortality and cardiovascular mortality (6).

SGLT2 (sodium-glucose cotransporter type 2) inhibitors are new glucose-lowering agents. As recently reported, they can reduce the risk of major adverse cardiovascular events (MACE) and improve renal outcomes.

Diabetes is also associated with an increased risk of adverse renal events so that diabetic kidney disease is the leading cause of the end-stage renal disease $(7,8)$. Moreover, renal events are likely to influence cardiovascular outcomes (9). The aim of this narrative review was to discuss the novel findings of the effects of SGLT2 inhibitors on cardiovascular and renal outcomes of type 2 diabetes. 


\section{Evidence Acquisition}

The literature published in PubMed, Scopus, Web of Science, Google Scholar, and Cochrane library was reviewed up to January 2019. The keywords including SGLT2 inhibitor, type 2 diabetes, cardiovascular effect, and renal effect were used in different combinations. We examined RCTs, observational studies, review articles, and systematic reviews.

\section{Results}

\subsection{Chemical Structure of SGLT2 Inhibitors}

Phlorizin, a C glucoside analog, is the first SGLT inhibitor with effects on the activity of both SGLT1 and SGLT2. It was first isolated from the root bark of apple trees in 1835 $(10,11)$. Then, the new C-aryl glucoside-derived SGLT2 inhibitors with non-hydrolysable C - C bond were discovered; thus, gliflozins are a novel class of glucose-lowering agents $(12-14)$.

Dapagliflozin was developed in 2008. The selectivity of dapagliflozin is about 1200 folds higher in humans for SGLT2 than for SGLT1 (15). Dapagliflozin was first approved in Europe in 2012, and the FDA approved it in 2014. Canagliflozin was approved by the FDA in 2013, with more than 400-fold higher inhibitory activity for SGLT2 than for SGLT1 (16).

The third agent in the gliflozin class is empagliflozin. European Medicines Agency (EMA) and the FDA approved empagliflozin in 2014. Empagliflozin has a higher selectivity for SGLT2 than for SGLT1 (2700 folds) (17). Another agent, ertugliflozin, was approved by EMA and FDA in 2017 (Table 1). In recent years, next-generation SGLT2 inhibitors, including ipragliflozin, tofogliflozin, and luseogliflozin, have been approved in Japan. Other agents that are in the late-phase of clinical trials include bexagliflozin and sotagliflozin (both as SGLT2/SGLT1 inhibitors) (18).

\subsection{Mechanism of Action}

The mechanism of glucose-lowering effects of SGLT2 inhibitors is the inhibition of glucose reabsorption in the proximal tubule of the kidney that leads to increased glucose excretion. The action of these agents is independent of insulin secretion (20). The SGLT2 expression is upregulated in hyperglycemia and the threshold for urinary glucose excretion is also higher (21). SGLT2 inhibitors usually lead to the excretion of 60 - 100 grams of glucose per day, leading to reduced glucose toxicity and insulin resistance (22). The glucose-lowering effect of SGLT2 inhibitors is glomerular filtration rate (GFR)-dependent, with an average of $0.79 \%$ reduction in HbA1c in normal renal function,

\begin{tabular}{|c|c|}
\hline Effects & SGLT2 Inhibitors \\
\hline Drugs & $\begin{array}{l}\text { Canagliflozin, dapagliflozin, empagliflozin, } \\
\text { ertuglifozin }\end{array}$ \\
\hline Target organ & The proximal tubule of the kidney \\
\hline Mechanism of action & $\begin{array}{l}\text { Forced glycosuria, Insulin-independent action, } \\
\text { Reduction in glucose toxicity }\end{array}$ \\
\hline Reduction in HbA1c & $0.7 \%$ to $1.0 \%$ \\
\hline Change in weight & Reduction \\
\hline Risk of hypoglycemia & Low \\
\hline Blood pressure & Reduction \\
\hline Use in renal impairment & Not effective in eGFR $<45 \mathrm{~mL} / \mathrm{min} / 1.73 \mathrm{~m}^{2 \mathrm{a}}$ \\
\hline Cardiovascular safety & $\begin{array}{l}\text { Proven (EMPA-REG OUTCOME, CANVAS, } \\
\text { DECLARE-TIMI) }\end{array}$ \\
\hline Side effects & $\begin{array}{l}\text { Genital infection, ketoacidosis, foot } \\
\text { amputation }^{\text {b }} \text {, fracture } \\
{ }^{c}\end{array}$ \\
\hline
\end{tabular}

Abbreviations: CANVAS, Canagliflozin Cardiovascular Assessment Study; eGFR, estimated glomerular filtration rate; EMPA-REG OUTCOME, empagliflozin cardiovascular OUTCOME events trial; DECLARE-TIMI, dapagliflozin effect on cardiovascular events-thrombolysis in myocardial infarction; HbA1c, glycated hemoglobin; SGLT2, sodium-glucose cotransporter type 2 .

${ }^{a}$ there was no need for dose adjustment in GFR between 45 and $60 \mathrm{~mL} / \mathrm{min} / 1.73$ $\mathrm{m}^{2}$ regarding empagliflozin. However, canagliflozin should be restricted to 100 $\mathrm{mg}$ per day in GFR between 45 and $60 \mathrm{~mL} / \mathrm{min} / 1.73 \mathrm{~m}^{2}$.

${ }^{\mathrm{b}}$ With canagliflozin, but it was not confirmed in real-world study.

With canagliflozin (19)

$0.3 \%-0.4 \%$ in the estimated GFR (eGFR) range of $30-59$ $\mathrm{mL} / \mathrm{min} / 1.73 \mathrm{~m}^{2}$, and no effect in the eGFR $<30 \mathrm{~mL} / \mathrm{min} / 1.73$ $\mathrm{m}^{2}$ (23). In the literature, a $0.7-1 \%$ reduction in $\mathrm{HbA1C}$ and $3-4 \mathrm{~kg}$ reduction in body weight were reported by using SGLT2 inhibitors (24). These agents also can lower blood pressure by modest diuresis and natriuresis (25).

\subsection{Cardiovascular Effects}

\subsubsection{Composite of Triple MACE}

Here, we briefly review the most recent trials of cardiovascular outcomes of SGLT2 inhibitors. The EMPA-REG OUTCOME study included 7020 participants with type $2 \mathrm{di}-$ abetes and previous cardiovascular disease (CVD) in two groups to receive empagliflozin or placebo added to standard treatment. Empagliflozin was associated with about $14 \%$ relative risk reduction in three-point MACE including cardiovascular death, non-fatal MI, and non-fatal stroke (P $=0.04$ for superiority) (26).

The CANVAS (Canagliflozin Cardiovascular Assessment Study) program included data of 10142 participants with type 2 diabetes and high risk for cardiovascular events from two trials (CANVAS and CANVAS-R: Canagliflozin Cardiovascular Assessment Study-Renal). The patients randomly received canagliflozin (100-300 $\mathrm{mg}$ ) or placebo and the mean follow-up time was 3.6 years. The rate of triple 
MACE was significantly lower in the canagliflozin treatment group than in the placebo group (hazard ratio (HR): 0.86; 95\% CI: 0.75 - 0.97; $\mathrm{P}=0.02$ for superiority) (27).

These findings were confirmed in two observational studies of the CVD-REAL Nordic study; the first one compared SGLT2 inhibitors (94\% with dapagliflozin) with other antidiabetic drugs (HR: 0.78; 95\% CI: 0.69 - 0.87; P < 0.001) (28) and the second one compared the newly treated patients with dapagliflozin versus newly treated patients with DPP-4 inhibitors (HR: 0.79; 95\% CI: $0.67-0.94 ; \mathrm{P}=$ $0.006)(29)$.

The result of a randomized controlled trial with dapagliflozin, the DECLARE-TIMI 58 study, has recently been published. The important point of this study, distinguishing it from other trials on SGLT2 inhibitors, was that the majority of the recruited patients had no previous ASCVD. In this trial, the researchers included 17160 patients including 10186 patients with no atherosclerotic cardiovascular disease. Patients received randomly either placebo or dapagliflozin. The results of composite primary endpoints showed noninferiority of dapagliflozin to placebo $(\mathrm{P}<0.001)$. The rate of MACE was $8.8 \%$ in dapagliflozin and 9.4\% in placebo groups (HR: 0.93; CI: $0.84-1.03$; $\mathrm{P}=0.17$ ) (30).

A meta-analysis of three large trials, including EMPAREG OUTCOME, CANVAS Program, and DECLARE-TIMI 58, was conducted by Zelniker et al. in 2018 revealing an 11\% reduction in MACE by using SGLT2 inhibitors (HR: 0.89; 95\% CI: $0.83-0.96, P=0.0014)$, and this effect only was seen in patients with previous atherosclerotic cardiovascular disease (HR: 0.86; 95\% CI: 0.80 - 0.93) (31).

$\mathrm{Wu}$ et al. in a meta-analysis of 57 published trials, including 33385 patients and six regulatory submissions (37525 patients) and data for seven different SGLT2 inhibitors, revealed a significant reduction in primary composite cardiovascular endpoints (MACE) in patients treated with SGLT2 inhibitors compared to placebo (relative risk: 0.84; 95\% CI: 0.75 - 0.95; $\mathrm{P}=0.006)(32)$.

A recently published meta-analysis by Zhang et al. in 2018 including five randomized controlled trials with 351476 participants showed a 20\% reduction in MACE with SGLT2 inhibitors (HR: 0.80; 95\% CI: $0.69-0.92 ; \mathrm{P}=0.002$ ) (19).

A meta-analysis by Tang et al. in 2016, including 37 trials and 29859 patients with diabetes, compared canagliflozin, dapagliflozin, and empagliflozin with placebo and other glucose-lowering treatments. The study showed that only was empagliflozin associated with a significantly lower risk of MACE compared to placebo (odds ratio (OR): 0.81; 95\% CI: 0.70 - 0.93) (33).

\subsubsection{Myocardial Infarction (Fatal or Non-Fatal)}

Data about myocardial infarction (MI) in patients treated with SGLT2 inhibitors are heterogeneous. In the EMPA-REG OUTCOME trial, there was no significant reduction in MI among patients with type 2 diabetes treated with empagliflozin 10 or $25 \mathrm{mg}$ compared to placebo (HR: 0.87; 95\% CI: 0.70 - 1.09; $\mathrm{P}=0.23)(26)$. Moreover, in the CANVAS trial, MI had not a significant reduction (HR: 0.85; 95\% CI: 0.69 -1.05; P > 0.05) (27), as the same as in two sub-studies using the CVD-REAL Nordic database $(28,29)$.

The sub-analysis of the CVD-REAL study showed a lower risk of MI with the initiation of SGLT2 inhibitors (HR: 0.85; 95\% CI: $0.72-1.00 ; P=0.05)$. Moreover, the CVD-REAL2 study, a large multinational study of patients with type 2 diabetes from six countries with over 400000 patients, confirmed this finding (HR: 0.81; 95\% CI: $0.74-0.88 ; \mathrm{P}<0.001)(34,35)$.

The rate of MI in the DECLARE-TIMI study was $4.6 \%$ in the dapagliflozin group and 5.1\% in the placebo group (HR: 0.89 ; 95\% CI: $0.77-1.01$ )(30). In a meta-analysis of 81 trials in 2016, SGLT2 inhibitors were not associated with lower risk of MI compared to placebo (OR: 0.89; 95\% CI: 0.74 - 1.09; P $=0.29$ ) (36). Zhang et al. in a meta-analysis showed that SGLT2 inhibitors significantly reduced the risk of non-fatal MI (HR: 0.86; 95\% CI: 0.76 - 0.98; P = 0.02) (19). Two metaanalysis by Savarese et al. and Monami et al. showed a significant reduction in MI (relative risk (RR): 0.803; 95\% CI: 0.668 - 0.965; $\mathrm{P}=0.02$ and HR: 0.77; 95\% CI: 0.63-0.94; $\mathrm{P}=$ 0.01 , respectively) $(37,38)$. Wu et al. in a meta-analysis of 57 randomized controlled trials demonstrated no significant reduction in MI (RR: 0.88; 95\% CI: 0.72-1.07; P = 0.18)(32).

\subsubsection{Stroke}

There are also heterogeneous findings about stroke in patients treated with this novel therapy. The EMPA-REG OUTCOME trial did not show a significant reduction in stroke in patients with type 2 diabetes treated with empagliflozin 10 or $25 \mathrm{mg}$ compared to placebo (HR:1.18; 95\% CI 0.89 - 1.56; $\mathrm{P}=0.26$ ) (26). Moreover, in CANAVAS trial, no reduction in stroke was observed (HR: 0.9; 95\% CI: 0.71-1.15; $\mathrm{P}=$ non-significant) (27), similar to two studies of CVD-REAL Nordic (HR: 0.86, 95\% CI: 0.72 - 1.04; P = 0.113 and HR: 0.79; 95\% CI: $0.61-1.03 ; \mathrm{P}=0.086)(37,38)$.

The DECLARE-TIMI study showed that ischemic stroke had no significant difference between the dapagliflozin group and the placebo group (HR: 1.01; 95\% CI: 0.84 - 1.21) (30). The CVD-REAL study showed a lower risk of stroke with the initiation of SGLT2 inhibitors in patients with diabetes (HR: 0.83; 95\% CI: $0.71-0.97 ; \mathrm{P}=0.05$ ). Moreover, the CVD-REAL2 study, a large multinational study of patients with type 2 diabetes, confirmed this finding (HR: 0.68; 95\% CI, 0.55 - 0.84; P $<0.001)(34,35)$. 
A meta-analysis by $\mathrm{Wu}$ et al. noted an adverse effect for non-fatal stroke (RR: 1.30; 95\% CI: 1.00 - 1.68; P = 0.049) (32). However, some meta-analysis studies showed no difference in the risk of stroke between SGLT2 inhibitors and placebo $(19,33,36-40)$.

\subsubsection{Heart Failure}

Heart failure is an important comorbidity in type 2 diabetes, especially in older patients (41). A significant reduction in hospitalization for heart failure was observed in patients with type 2 diabetes treated with empagliflozin in the EMPA-REG OUTCOME trial (HR: 0.65; 95\% CI: 0.50 $0.85 ; \mathrm{P}=0.002)(26)$. This effect was noticed rapidly in the first six months of the initiation of treatment (42); however, in CANVAS trial, there was no significant reduction in hospitalization for heart failure (27). A systemic review and meta-analysis with trial sequential analysis by Zhang et al. in 2018 demonstrated a significant reduction in hospitalization for heart failure in patients receiving SGLT2 inhibitors (HR: 0.62; 95\% CI: 0.55 - 0.69; P $<0.001$ ) (19).

In patients treated with dapagliflozin in the DECLARE trial, the reduction rate of hospitalization for heart failure was the only cardiovascular outcome that showed superiority (HR: 0.73; 95\% CI: 0.61-0.88) (30).

Zelniker et al. conducted a meta-analysis of three large trials of SGLT2 inhibitors, including EMPA-REG OUTCOME, CANVAS Program, and DECLARE-TIMI 58, and showed a 23\% reduction in the risk of cardiovascular death or hospitalization for heart failure (HR: 0.77; 95\% CI: $0.71-0.84$, P $<0.0001)$ and this benefit was the same in patients with and without cardiovascular disease and patients with and without previous heart failure (31).

Moreover, the meta-analyses of trials by Savarese et al. and Saad et al. showed a significant reduction in hospitalization due to heart failure (HR: 0.652; 95\% CI: 0.517-0.823; $\mathrm{P}<0.001$ and HR: 0.67; 95\% CI, $0.51-0.87$; $=0.003$, respectively) $(36,37)$; however, some meta-analyses of studies with the exclusion of CANVAS program and EMPA-REG OUTCOME did not show a reduction in hospitalization for heart failure $(19,37,39,40)$.

The CVD-REAL study of 309056 diabetic patients newly treated with SGLT2 inhibitors or other antidiabetic drugs in the United States, Norway, Denmark, Sweden, and the United Kingdom showed the real-world effectiveness of the initiation of SGLT2 inhibitors in reduced hospitalization due to heart failure (HR: 0.61; 95\% CI: 0.51 - 0.73; P < 0.001) (43). Moreover, the CVD-REAL2 study, a large multinational study of patients with type 2 diabetes, confirmed this finding (HR: 0.64; 95\% CI: $0.50-0.82 ; \mathrm{P}=0.001$ ) (35), in line with the two studies of CVD-REAL Nordic (HR: 0.7; 95\% CI: 0.7261-0.81; P > 0.001 and HR: 0.62; 95\% CI: 0.50$0.77 ; \mathrm{P}<0.001)(29,32)$. In a meta-analysis of 37 trials in
2016, only was empagliflozin associated with the lower incidence of heart failure or hospitalization due to heart failure (OR: 0.65; 95\% CI: 0.50 - 0.84) (33).

\subsubsection{Cardiovascular Mortality}

Cardiovascular mortality rate was lower in patients with type 2 diabetes treated with SGLT2 inhibitors in the EMPA-REG OUTCOME trial (38\% relative risk reduction)(HR: 0.62; 95\% CI: 0.49 - 0.77; P < 0.001) (26). A similar finding was obtained in the observational CVD-REAL Nordic study that compared SGLT2 inhibitors with other antidiabetic agents (HR: 0.53; 95\% CI: 0.40 - 0.71; P < 0.001) (28). Some meta-analyses also showed a reduction in cardiovascular mortality in patients receiving SGLT2 inhibitors (32, 36-38).

The reduction in the cardiovascular mortality rate was not significant in CANVAS program comparing canagliflozin with placebo (HR: 0.87; 95\% CI: 0.72 - 1.06), in the DECLARE-TIMI trial comparing dapagliflozin with placebo (HR: 0.98; 95\% CI: 0.82 - 1.17), and in the observational CVD-REAL Nordic study comparing dapagliflozin 10 mg with DPP4 inhibitors (HR: 0.76; 95\% CI: 0.53-1.08; P $=0.122)(27,29,30)$. In a meta-analysis by Zhang et al. in 2018, there was a significant reduction in cardiovascular mortality in patients treated with SGLT2 inhibitors (HR: 0.77; 95\% CI, 0.60 - 0.98; P = 0.033) (19).

\subsubsection{All-Cause Mortality}

A significant reduction in all-cause mortality was shown in patients receiving SGLT2 inhibitors compared to placebo in the EMPA-REG OUTCOME trial (32\% relative risk reduction) (26). However, there was no significant reduction in all-cause mortality in CANVAS trial in patient receiving canagliflozin versus placebo (HR: 0.87; 95\% CI: 0.74 - 1.01; $\mathrm{P}=0.033$ ) (27). Conducted by Tang et al., a meta-analysis of 37 trials involving 29859 patients compared canagliflozin, empagliflozin, and dapagliflozin with placebo and other glucose-lowering agents and demonstrated that empagliflozin was the only treatment lowering the risk of all-cause mortality (OR: 0.67; 95\% CI: 0.560.81 ), indicating a protective effect (33).

In the DECLARE-TIMI trial, death from any cause was not significantly different between the dapagliflozin and placebo groups (6.2\% in dapagliflozin and $6.6 \%$ in placebo; HR: 0.93; 95\% CI: 0.82-1.04)(30).

Some other meta-analyses showed that SGLT2 inhibitors significantly reduced the risk of all-cause mortality (19, 32, 36-38). Moreover, observational studies, including CVD-REAL, CVD-REAL2, and CVD-REAL Nordic, confirmed these findings $(28,29,35,43)$. 


\subsubsection{Mechanisms of Cardiovascular Effects}

Several mechanisms have been proposed for cardiovascular effects of SGLT2 inhibitors, including osmotic diuresis and natriuresis leading to blood pressure lowering, decrease in arterial stiffness and vascular resistance, decrease in weight, and decrease in uric acid and oxidative stress. Increases in hemoglobin and hematocrit may also play a role. The EMPA REG trial showed a strong association between hemoglobin or hematocrit and reduced heart failure and the risk of death $(26,44-46)$. Glucagon regulates cardiac glucose utilization and has a positive inotropic and anti-arrhythmogenic effects (47). Empagliflozin can increase blood glucagon levels, possibly due to glucose excretion and/or exerting a direct effect on pancreatic alpha cells. The reduced risks of heart failure and cardiovascular mortality may be explained by the improvement of arrhythmia and myocardial function related to higher levels of glucagon (48-50). A recent hypothesis suggests that SGLT2 inhibitors may improve myocardial work efficiency by changing the fuel metabolism from free fatty acids to ketones, which are more efficient energetic fuels (51).

Another new theory suggests the reduction of cardiovascular mortality with empagliflozin may be due to a direct effect on cardiomyocytes to improve myocardial function and reduce rhythm disturbances. A weak inhibitory effect of empagliflozin on myocardial SGLT1, particularly in patients with overexpression of SGLT1 after ischemia, could contribute to the reduction of cardiovascular mortality (52). The cardiovascular benefits of SGLT2 inhibitors and probable mechanisms of these actions are summarized in Table 2 and Figure 1, respectively.

\subsection{Renal Effects of SGLT2 Inhibitors}

\subsubsection{Reduction in the Progression of Albuminuria}

A significant relative risk reduction in progression to macroalbuminuria was observed in the empagliflozin group versus the placebo group in the EMPA-REG OUTCOME trial. It was shown that $11.2 \%$ of the patients in the empagliflozin group had progression to macroalbuminuria compared to $16.2 \%$ in the placebo group, with a $38 \%$ relative risk reduction (53). The CANVAS program showed a $27 \%$ reduction in the progression of albuminuria and also more regression of albuminuria compare to placebo (HR: 0.73; 95\% CI: $0.67-0.79$ and HR:1.70; 95\% CI:1.51-1.91, respectively) (27).

A recent meta-analysis in 2018 by Seidu et al. including 40 randomized clinical trials revealed that the administration of SGLT2 inhibitors, particularly canagliflozin and empagliflozin, to patients with or without renal failure improved albuminuria and slowed the rate of progression to macroalbuminuria (54).

\subsubsection{Reduction in Doubling of the Serum Creatinine}

In the EMPA-REG OUTCOME trial, doubling of the serum creatinine level occurred in $1.5 \%$ of participants treated with empagliflozin compared to $2.3 \%$ in participants receiving placebo, with a $44 \%$ risk reduction (HR 0.56; 95\% CI: 0.39 - 0.79; P< 0.001) (53).

A meta-analysis by Seidu et al. showed treatment with SGLT2 inhibitors was associated with initial increases in serum creatinine levels, followed by a return to baseline in patients with renal failure, but serum creatinine was preserved in those without renal failure (54).

\subsubsection{The Lower Rate of Renal Replacement Therapy}

In end-stage renal disease, patients require renal replacement therapy including dialysis or kidney transplant (55). In the EMPA-REG OUTCOME trial, the renal replacement therapy started in $0.3 \%$ of patients receiving empagliflozin compared to $0.6 \%$ in those receiving placebo, with a significant risk reduction of $55 \%$ in patients treated with empagliflozin (HR: 0.45; 95\% CI: 0.21 - 0.97; P = 0.04) (53).

CANVAS program showed a $40 \%$ reduction in the composite renal outcome, including the requirement for kidney replacement therapy, eGFR, or mortality from renal causes (HR: 0.60; 95\% CI: 0.47 - 0.77) (27).

\subsubsection{Effects on Renal Function Over Time}

In the EMPA-REG OUTCOME trial, an initial reduction occurred in the eGFR in the empagliflozin group. In the first four weeks, the eGFR decreased in the empagliflozin users, weekly decreases of $0.62 \pm 0.04 \mathrm{~mL} / \mathrm{min} / 1.73 \mathrm{~m}^{2}$ in patients treated with empagliflozin $10 \mathrm{mg}$ and $0.82 \pm$ $0.04 \mathrm{~mL} / \mathrm{min} / 1.73 \mathrm{~m}^{2}$ in patients treated with empagliflozin $25 \mathrm{mg}$, compared to a small increase of GFR (0.01 \pm $0.04 \mathrm{~mL} / \mathrm{min} / 1.73 \mathrm{~m}^{2}$ ) in patients receiving placebo. In a long-term follow-up, the eGFR remained stable in the empagliflozin group, but declined in patients receiving a placebo; the annual decreases of $0.19 \pm 0.11 \mathrm{~mL} / \mathrm{min} / 1.73$ $\mathrm{m}^{2}$ in empagliflozin users and a reduction of $1.67 \pm 0.13$ $\mathrm{mL} / \mathrm{min} / 1.73 \mathrm{~m}^{2}$ in placebo users were noted $(\mathrm{P}<0.001)$ (53).

In the CANVAS program, after a decrease in the eGFR with canagliflozin ( 76 to $73 \mathrm{~mL} / \mathrm{min} / 1.73 \mathrm{~m}^{2}$ ) at three months, it remained stable for six years while the eGFR gradually declined with placebo (19).

In populations with renal impairment, SGLT2 inhibition was associated with an initial decline in the eGFR, followed by a return to baseline. In patients without renal impairment, an analysis of 17 studies showed no significant change in the eGFR with SGLT2 inhibitors compared to placebo $\left(\right.$ mean difference $=0.51 \mathrm{~mL} / \mathrm{min} / 1.73 \mathrm{~m}^{2} ; \mathrm{P}=0.403$ ) (54). 


\begin{tabular}{|c|c|c|c|}
\hline Outcomes & EMPA-REG Trial & CANVAS Program & DECLARE - TIMI \\
\hline Three-point MACE & $0.86(0.74-0.99)$ & $0.86(0.75-0.97)$ & $0.93(0.84-1.03)$ \\
\hline Myocardial infarction & $0.87(0.70-1.09)$ & $0.89(0.73-1.09)$ & $0.89(0.77-1.01)$ \\
\hline Stroke & $1.18(0.89-1.56)$ & $0.87(0.69-1.09)$ & $1.01(0.84-1.21)$ \\
\hline Hospitalization for heart failure & $0.65(0.5-0.85)$ & $0.67(0.52-0.87)$ & $0.73(0.61-0.88)$ \\
\hline Cardiovascular mortality & $0.62(0.49-0.77)$ & $0.87(0.72-1.06)$ & $0.98(0.82-1.17)$ \\
\hline All-cause mortality & $0.61(0.53-0.70)$ & $0.87(0.74-1.01)$ & $0.93(0.82-1.04)$ \\
\hline
\end{tabular}

Abbreviations: CI, confidence interval; MACE, major adverse cardiovascular events.

${ }^{\mathrm{a}}$ Values are expressed as hazard ratio $(\mathrm{CI})$.

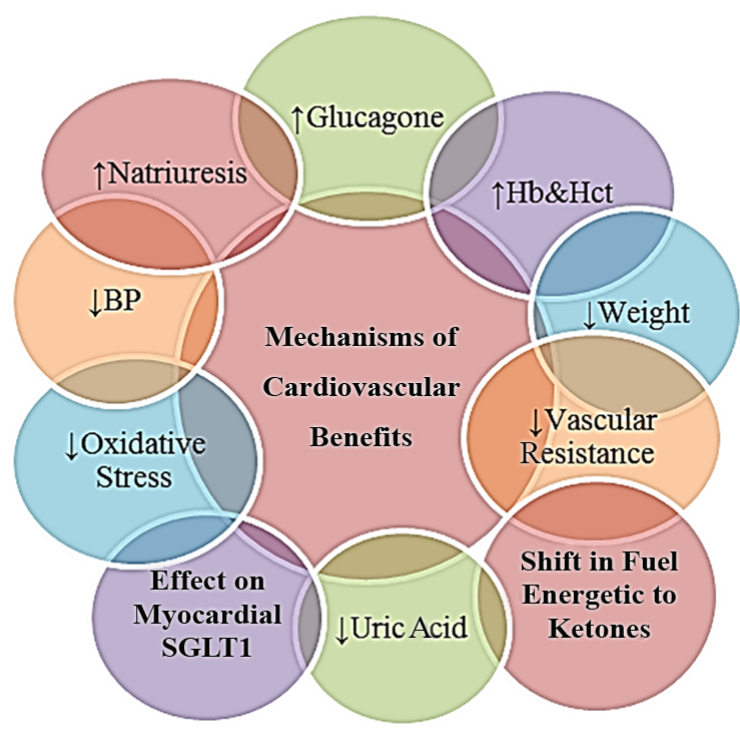

Figure 1. Probable mechanisms of cardiovascular benefits of SGLT2 inhibitors. Hb, hemoglobin; Hct, hematocrit; BP, blood pressure

In the DECLARE-TIMI trial, the composite renal outcome, including a $\geq 40 \%$ decrease in eGFR to lower than $60 \mathrm{~mL} / \mathrm{min} / 1.73 \mathrm{~m}^{2}$, decrease in end-stage renal disease, or decrease in death from renal causes, was improved in the dapagliflozin group than in the placebo group (HR: 0.53; 95\% CI: 0.43 - 0.66)(30).

The meta-analysis of three trials, including EMPA-REG OUTCOME, CANVAS Program, and DECLARE-TIMI 58, revealed a $45 \%$ reduction in the risk of progression of renal disease (HR: $0.55 ; 95 \%$ CI: $0.48-0.64, \mathrm{P}<0.0001$ ) and this benefit was similar in patients with and without atherosclerotic cardiovascular disease (31).

\subsubsection{Mechanisms of Renal Effects of SGLT2 Inhibitors}

The renal protection mechanisms are multifactorial. SGLT2 inhibitors reduce the reabsorption of sodium in the proximal tubule of the kidney and increase the delivery of sodium to the macula densa; this may restore the tubular glomerular feedback, leading to a reduction in the kidney blood flow, a decrease in glomerular hyperfiltration, and a reduction in intra-glomerular pressure (44). These effects may result in an acute decrease in albuminuria and eGFR, followed by eGFR stability in the long run (56).

As previously discussed, SGLT2 inhibitors have beneficial effects on multiple risk factors of renal impairment, including high blood glucose, high blood pressure, high serum uric acid, and body weight (23). These suggest the potential nephroprotective effect of SGLT2 inhibitors in patients with diabetes. The renal effects of SGLT2 inhibitors are summarized in Table 3. 


\begin{tabular}{|c|c|c|c|}
\hline Renal Outcome & $\begin{array}{c}\text { EMPA REG Trial Related Risk } \\
\text { Reduction, } \%\end{array}$ & $\begin{array}{c}\text { CANVAS Program Related Risk } \\
\text { Reduction, } \%\end{array}$ & DECLARE-TIMI Risk Reduction, \% \\
\hline Progression of albuminuria & 38 & 27 & NA \\
\hline Doubling of serum creatinine & 44 & NA & NA \\
\hline $\begin{array}{l}\text { The rate of renal replacement } \\
\text { therapy }\end{array}$ & 55 & $40^{\mathrm{a}}$ & NA \\
\hline Effects on renal function & $\begin{array}{l}\text { A short-term decrease in eGFR in the } \\
\text { first four weeks, followed by eGFR } \\
\text { stability in the long-term follow-up }\end{array}$ & $\begin{array}{l}\text { A decrease in eGFR at three months, } \\
\text { followed by stability in a six-year } \\
\text { follow-up }\end{array}$ & NA \\
\hline Composite renal outcome $^{a}$ & NA & 40 & $47^{\mathrm{b}}$ \\
\hline
\end{tabular}

Abbreviation: NA, not available.

${ }^{a}$ Composite renal outcome: reduction in the need for renal replacement therapy, reduction in estimated glomerular filtration rate (eGFR), and reduction in death from renal causes $(15,19)$.

${ }^{\mathrm{b}}$ The renal composite outcome including $\geq 40 \%$ decrease in eGFR to $<60 \mathrm{~mL} / \mathrm{min} / 1.73 \mathrm{~m}^{2}$, decrease in end-stage renal disease, or decrease in death from renal causes.

\section{Conclusions}

Given the suboptimal glycemic and the importance of cardiovascular and renal risk reduction in type 2 diabetes and the high burden of cardiovascular and renal disease in patients with diabetes, novel therapies such as SGLT2 inhibitors seem to have an important clinical advantage to improve glycemic control and cardiovascular and renal outcomes.

\section{Footnotes}

Authors' Contribution: Alireza Esteghamati developed the original idea, gathered data, and revised the manuscript. Soghra Rabizadeh gathered data and wrote the manuscript. Manouchehr Nakhjavani revised the manuscript.

Conflicts of Interests: The authors have no conflicts of interests.

Funding/Support: There was no funding for this study.

\section{References}

1. Farkouh ME, Boden WE, Bittner V, Muratov V, Hartigan P, Ogdie M, et al. Risk factor control for coronary artery disease secondary prevention in large randomized trials. J Am Coll Cardiol. 2013;61(15):1607-15. doi: 10.1016/j.jacc.2013.01.044. [PubMed: 23500281].

2. Saydah SH, Fradkin J, Cowie CC. Poor control of risk factors for vascular disease among adults with previously diagnosed diabetes. JAMA. 2004;291(3):335-42. doi: 10.1001/jama.291.3.335. [PubMed: 14734596].

3. Emerging Risk Factors Collaboration, Sarwar N, Gao P, Seshasai SR, Gobin R, Kaptoge S, et al. Diabetes mellitus, fasting blood glucose concentration, and risk of vascular disease: A collaborative metaanalysis of 102 prospective studies. Lancet.2010;375(9733):2215-22. doi: 10.1016/s0140-6736(10)60484-9. [PubMed: 20609967]. [PubMed Central: PMC2904878].

4. Mukamal KJ, Nesto RW, Cohen MC, Muller JE, Maclure M, Sherwood $\mathrm{JB}$, et al. Impact of diabetes on long-term survival after acute myocardial infarction: Comparability of risk with prior myocardial infarction. Diabetes Care. 2001;24(8):1422-7. doi: 10.2337/diacare.24.8.1422. [PubMed: 11473080].
5. Alemi H, Khaloo P, Mansournia MA, Rabizadeh S, Salehi SS, Mirmiranpour $\mathrm{H}$, et al. Pulse pressure and diabetes treatments: Blood pressure and pulse pressure difference among glucose lowering modality groups in type 2 diabetes. Medicine (Baltimore). 2018;97(6). e9791. doi: 10.1097/MD.0000000000009791. [PubMed: 29419672]. [PubMed Central: PMC5944681].

6. Raee MR, Nargesi AA, Heidari B, Mansournia MA, Larry M, Rabizadeh S, et al. All-cause and cardiovascular mortality following treatment with metformin or glyburide in patients with type 2 diabetes mellitus. Arch Iran Med. 2017;20(3):141-6. [PubMed: 28287807].

7. Rabizadeh S, Dehghani Firouzabadi F, Noshad S, Esteghamati S, Afarideh M, Ghajar A, et al. Beneficial effects of pentoxifylline plus losartan dual therapy in type 2 diabetes with nephropathy. Am J Med Sci. 2018;355(5):442-8. doi: 10.1016/j.amjms.2017.12.015. [PubMed: 29753374].

8. Esteghamati A, Larijani B, Aghajani MH, Ghaemi F, Kermanchi J, Shahrami A, et al. Diabetes in Iran: Prospective analysis from first nationwide diabetes report of national program for prevention and control of diabetes (NPPCD-2016). Sci Rep. 2017;7(1):13461. doi: 10.1038/s41598-017-13379-z. [PubMed: 29044139]. [PubMed Central: PMC5647418].

9. Wang Y, Katzmarzyk PT, Horswell R, Zhao W, Johnson J, Hu G. Kidney function and the risk of cardiovascular disease in patients with type 2 diabetes. Kidney Int. 2014;85(5):1192-9. doi: 10.1038/ki.2013.396. [PubMed: 24107845]. [PubMed Central: PMC4141539].

10. Link JT, Sorensen BK. A method for preparing C-glycosides related to phlorizin. Tetrahedron Letters. 2000;41(48):9213-7. doi: 10.1016/s00404039(00)01709-3.

11. Ehrenkranz JR, Lewis NG, Kahn CR, Roth J. Phlorizin: A review. Diabetes Metab Res Rev. 2005;21(1):31-8. doi: 10.1002/dmrr.532. [PubMed: 15624123].

12. Larson GL. The synthesis of gliflozins. Chem Today. 2015;33:37-40.

13. Brito-Arias M. Synthesis and characterization of glycosides. Springer; 2007.

14. Satirapoj B. Sodium-glucose cotransporter 2 inhibitors with renoprotective effects. Kidney Dis (Basel). 2017;3(1):24-32. doi: 10.1159/000471765. [PubMed: 28785561]. [PubMed Central: PMC5527177].

15. Meng W, Ellsworth BA, Nirschl AA, McCann PJ, Patel M, Girotra RN, et al. Discovery of dapagliflozin: A potent, selective renal sodium-dependent glucose cotransporter 2 (SGLT2) inhibitor for the treatment of type 2 diabetes. J Med Chem. 2008;51(5):1145-9. doi: 10.1021/jm701272q. [PubMed: 18260618]. 
16. Nomura S, Sakamaki S, Hongu M, Kawanishi E, Koga Y, Sakamoto T, et al. Discovery of canagliflozin, a novel C-glucoside with thiophene ring, as sodium-dependent glucose cotransporter 2 inhibitor for the treatment of type 2 diabetes mellitus. J Med Chem. 2010;53(17):635560. doi: 10.1021/jm100332n. [PubMed: 20690635].

17. Grempler R, Thomas L, Eckhardt M, Himmelsbach F, Sauer A, Sharp DE, et al. Empagliflozin, a novel selective sodium glucose cotransporter-2 (SGLT-2) inhibitor: Characterisation and comparison with other SGLT2 inhibitors. Diabetes Obes Metab. 2012;14(1):83-90. doi: 10.1111/j.14631326.2011.01517.x. [PubMed: 21985634].

18. Anderson KS, LaBaer J. The sentinel within: Exploiting the immune system for cancer biomarkers. J Proteome Res. 2005;4(4):112333. doi: 10.1021/pr0500814. [PubMed: 16083262]. [PubMed Central: PMC2522321].

19. Zhang XL, Zhu QQ, Chen YH, Li XL, Chen F, Huang JA, et al. Cardiovascular safety, long-term noncardiovascular safety, and efficacy of sodium-glucose cotransporter 2 inhibitors in patients with type 2 diabetes mellitus: A systemic review and meta-analysis with trial sequential analysis. J Am Heart Assoc. 2018;7(2). doi: 10.1161/JAHA.117.007165. [PubMed: 29353233]. [PubMed Central: PMC5850151].

20. Marsenic O. Glucose control by the kidney: An emerging target in diabetes. Am J Kidney Dis. 2009;53(5):875-83. doi: 10.1053/j.ajkd.2008.12.031. [PubMed: 19324482].

21. Zhao Y, Gao P, Sun F, Li Q, Chen J, Yu H, et al. Sodium intake regulates glucose Homeostasis through the PPARdelta/adiponectinmediated SGLT2 Pathway. Cell Metab. 2016;23(4):699-711. doi: 10.1016/j.cmet.2016.02.019. [PubMed: 27053360].

22. Tanaka A, Node K. Emerging roles of sodium-glucose cotransporter 2 inhibitors in cardiology. J Cardiol. 2017;69(3):501-7. doi: 10.1016/j.jjcc.2016.10.019. [PubMed: 28043708]

23. van Bommel EJ, Muskiet MH, Tonneijck L, Kramer MH, Nieuwdorp M, van Raalte DH. SGLT2 inhibition in the diabetic kidney-from mechanisms to clinical outcome. Clin J Am Soc Nephrol. 2017;12(4):700-10. doi: 10.2215/CJN.06080616. [PubMed: 28254770]. [PubMed Central: PMC5383382]

24. Inzucchi SE, Zinman B, Wanner C, Ferrari R, Fitchett D, Hantel S, et al. SGLT-2 inhibitors and cardiovascular risk: Proposed pathways and review of ongoing outcome trials. Diab Vasc Dis Res. 2015;12(2):90-100. doi: 10.1177/1479164114559852. [PubMed: 25589482]. [PubMed Central: PMC4361459].

25. Powell DR, DaCosta CM, Gay J, Ding ZM, Smith M, Greer J, et al. Improved glycemic control in mice lacking Sglt1 and Sglt2. Am J Physiol Endocrinol Metab. 2013;304(2):E117-30. doi: 10.1152/ajpendo.00439.2012. [PubMed: 23149623].

26. Zinman B, Wanner C, Lachin JM, Fitchett D, Bluhmki E, Hantel S, et al. Empagliflozin, cardiovascular outcomes, and mortality in type 2 diabetes. N Engl J Med. 2015;373(22):2117-28. doi: 10.1056/NEJMoa1504720. [PubMed: 26378978].

27. Neal B, Perkovic V, Mahaffey KW, de Zeeuw D, Fulcher G, Erondu N, et al. Canagliflozin and cardiovascular and renal events in type $2 \mathrm{di}$ abetes. N Engl J Med. 2017;377(7):644-57. doi: 10.1056/NEJMoa1611925. [PubMed: 28605608].

28. Birkeland KI, Jorgensen ME, Carstensen B, Persson F, Gulseth HL, Thuresson M, et al. Cardiovascular mortality and morbidity in patients with type 2 diabetes following initiation of sodium-glucose cotransporter-2 inhibitors versus other glucose-lowering drugs (CVDREAL Nordic): A multinational observational analysis. Lancet Diabetes Endocrinol. 2017;5(9):709-17. doi: 10.1016/S2213-8587(17)30258-9. [PubMed: 28781064].

29. Persson F, Nystrom T, Jorgensen ME, Carstensen B, Gulseth HL, Thuresson $\mathrm{M}$, et al. Dapagliflozin is associated with lower risk of cardiovascular events and all-cause mortality in people with type 2 diabetes (CVD-REAL Nordic) when compared with dipeptidyl peptidase-4 inhibitor therapy: A multinational observational study. Diabetes Obes Metab. 2018;20(2):344-51. doi: 10.1111/dom.13077. [PubMed: 28771923]. [PubMed Central: PMC5811811].
30. Wiviott SD, Raz I, Bonaca MP, Mosenzon O, Kato ET, Cahn A, et al Dapagliflozin and cardiovascular outcomes in type 2 diabetes. $N$ Engl J Med. 2019;380(4):347-57. doi: 10.1056/NEJMoa1812389. [PubMed: 30415602].

31. Zelniker TA, Wiviott SD, Raz I, Im K, Goodrich EL, Bonaca MP, et al. SGLT2 inhibitors for primary and secondary prevention of cardiovascular and renal outcomes in type 2 diabetes: A systematic review and meta-analysis of cardiovascular outcome trials. Lancet. 2019;393(10166):31-9. doi: 10.1016/S0140-6736(18)32590-X. [PubMed: 30424892].

32. Wu JH, Foote C, Blomster J, Toyama T, Perkovic V, Sundstrom J, et al. Effects of sodium-glucose cotransporter-2 inhibitors on cardiovascular events, death, and major safety outcomes in adults with type 2 diabetes: A systematic review and meta-analysis. Lancet Diabetes Endocrinol. 2016;4(5):411-9. doi:10.1016/S2213-8587(16)00052-8. [PubMed: 27009625].

33. Tang H, Fang Z, Wang T, Cui W, Zhai S, Song Y. Meta-analysis of effects of sodium-glucose cotransporter 2 inhibitors on cardiovascular outcomes and all-cause mortality among patients with type 2 diabetes mellitus. Am J Cardiol. 2016;118(11):1774-80. doi: 10.1016/j.amjcard.2016.08.061. [PubMed: 27666177].

34. Kosiborod M, Birkeland KI, Cavender MA, Fu AZ, Wilding JP, Khunti $\mathrm{K}$, et al. Rates of myocardial infarction and stroke in patients initiating treatment with SGLT2-inhibitors versus other glucose-lowering agents in real-world clinical practice: Results from the CVD-REAL study. Diabetes Obes Metab. 2018;20(8):1983-7. doi: 10.1111/dom.13299. [PubMed: 29569378]. [PubMed Central: PMC6055705].

35. Kosiborod M, Lam CSP, Kohsaka S, Kim DJ, Karasik A, Shaw J, et al Cardiovascular events associated with SGLT-2 inhibitors versus other glucose-lowering drugs. J Am Coll Cardiol. 2018;71(23):2628-39. doi: 10.1016/j.jacc.2018.03.009. [PubMed: 29540325].

36. Saad M, Mahmoud AN, Elgendy IY, Abuzaid A, Barakat AF, Elgendy AY, et al. Cardiovascular outcomes with sodium-glucose cotransporter-2 inhibitors in patients with type II diabetes mellitus: A meta-analysis of placebo-controlled randomized trials. Int J Cardiol. 2017;228:352-8. doi: 10.1016/j.ijcard.2016.11.181. [PubMed: 27866027].

37. Savarese G, D'Amore C, Federici M, De Martino F, Dellegrottaglie S, Marciano C, et al. Effects of dipeptidyl peptidase 4 inhibitors and sodium-glucose linked cotransporter-2 Inhibitors on cardiovascular events in patients with type 2 diabetes mellitus: A meta-analysis. Int J Cardiol. 2016;220:595-601. doi: 10.1016/j.ijcard.2016.06.208. [PubMed: 27390996].

38. Monami M, Dicembrini I, Mannucci E. Effects of SGLT-2 inhibitors on mortality and cardiovascular events: A comprehensive meta-analysis of randomized controlled trials. Acta Diabetol. 2017;54(1):19-36. doi: 10.1007/s00592-016-0892-7. [PubMed: 27488726].

39. Sonesson C, Johansson PA, Johnsson E, Gause-Nilsson I. Cardiovascular effects of dapagliflozin in patients with type 2 diabetes and different risk categories: A meta-analysis. Cardiovasc Diabetol. 2016;15:37. doi: 10.1186/s12933-016-0356-y. [PubMed: 26895767]. [PubMed Central: PMC4761166].

40. Salsali A, Kim G, Woerle HJ, Broedl UC, Hantel S. Cardiovascular safety of empagliflozin in patients with type 2 diabetes: A metaanalysis of data from randomized placebo-controlled trials. Diabetes Obes Metab. 2016;18(10):1034-40. doi:10.1111/dom.12734. [PubMed: 27376831]. [PubMed Central: PMC5096016].

41. Bertoni AG, Hundley WG, Massing MW, Bonds DE, Burke GL, Goff DJ. Heart failure prevalence, incidence, and mortality in the elderly with diabetes. Diabetes Care. 2004;27(3):699-703. doi: 10.2337/diacare.27.3.699. [PubMed: 14988288].

42. Sattar N, McLaren J, Kristensen SL, Preiss D, McMurray JJ. SGLT2 Inhibition and cardiovascular events: Why did EMPA-REG outcomes surprise and what were the likely mechanisms? Diabetologia. 2016;59(7):1333-9. doi: 10.1007/s00125-016-3956-x. [PubMed: 27112340]. [PubMed Central: PMC4901113]. 
43. Kosiborod M, Cavender MA, Fu AZ, Wilding JP, Khunti K, Holl RW, et al. Lower risk of heart failure and death in patients initiated on sodiumglucose cotransporter-2 inhibitors versus other glucose-lowering drugs: The CVD-REAL study (comparative effectiveness of cardiovascular outcomes in new users of sodium-glucose cotransporter-2 inhibitors). Circulation. 2017;136(3):249-59. doi: 10.1161/CIRCULATIONAHA.117.029190. [PubMed: 28522450]. [PubMed Central: PMC5515629].

44. Cherney DZ, Perkins BA, Soleymanlou N, Har R, Fagan N, Johansen OE, et al. The effect of empagliflozin on arterial stiffness and heart rate variability in subjects with uncomplicated type 1 diabetes mellitus. Cardiovasc Diabetol. 2014;13:28. doi: 10.1186/1475-2840-13-28. [PubMed: 24475922]. [PubMed Central: PMC3915232].

45. Neeland IJ, McGuire DK, Chilton R, Crowe S, Lund SS, Woerle HJ, et al. Empagliflozin reduces body weight and indices of adipose distribution in patients with type 2 diabetes mellitus. Diab Vasc Dis Res. 2016;13(2):119-26. doi: 10.1177/1479164115616901. [PubMed: 26873905]. [PubMed Central: PMC4768401].

46. Cheeseman C. Solute carrier family 2, member 9 and uric acid homeostasis. Curr Opin Nephrol Hypertens. 2009;18(5):428-32. doi: 10.1097/MNH.ob013e32832ee3de. [PubMed: 19593129].

47. Jones BJ, Tan T, Bloom SR. Minireview: Glucagon in stress and energy homeostasis. Endocrinology. 2012;153(3):1049-54. doi: 10.1210/en.20111979. [PubMed: 22294753]. [PubMed Central: PMC3281544].

48. Ferrannini E, Muscelli E, Frascerra S, Baldi S, Mari A, Heise T, et al. Metabolic response to sodium-glucose cotransporter 2 inhibition in type 2 diabetic patients. J Clin Invest. 2014;124(2):499508. doi: 10.1172/JCI72227. [PubMed: 24463454]. [PubMed Central: PMC3904627].

49. Bonner C, Kerr-Conte J, Gmyr V, Queniat G, Moerman E, Thevenet J, et al. Inhibition of the glucose transporter SGLT2 with dapagliflozin in pancreatic alpha cells triggers glucagon secretion. Nat Med. 2015;21(5):512-7. doi: 10.1038/nm.3828. [PubMed: 25894829].
50. Ceriello A, Genovese S, Mannucci E, Gronda E. Glucagon and heart in type 2 diabetes: New perspectives. Cardiovasc Diabetol. 2016;15(1):123. doi: 10.1186/s12933-016-0440-3. [PubMed: 27568179]. [PubMed Central: PMC5002329].

51. Cavaiola TS, Pettus J. Cardiovascular effects of sodium glucose cotransporter 2 inhibitors. Diabetes Metab Syndr Obes. 2018;11:133-48. doi: 10.2147/DMSO.S154602. [PubMed: 29695924]. [PubMed Central: PMC5905845].

52. Luconi M, Raimondi L, Di Franco A, Mannucci E. Which is the main molecular target responsible for the cardiovascular benefits in the EMPA-REG OUTCOME trial? A journey through the kidney, the heart and other interesting places. Nutr Metab Cardiovasc Dis. 2016;26(12):1071-8. doi: 10.1016/j.numecd.2016.09.001. [PubMed: 27776917].

53. Warner MR. Age incidence and site distribution of mammary dysplasias in young beagle bitches. J Natl Cancer Inst. 1976;57(1):57-61. doi: 10.1093/jnci/57.1.57. [PubMed: 1034018].

54. Seidu S, Kunutsor SK, Cos X, Gillani S, Khunti K; For, et al. SGLT2 inhibitors and renal outcomes in type 2 diabetes with or without renal impairment: A systematic review and meta-analysis. Prim Care Diabetes. 2018;12(3):265-83. doi: 10.1016/j.pcd.2018.02.001. [PubMed: 29482993].

55. Gross JL, de Azevedo MJ, Silveiro SP, Canani LH, Caramori ML, Zelmanovitz T. Diabetic nephropathy: Diagnosis, prevention, and treatment. Diabetes Care. 2005;28(1):164-76. doi: 10.2337/diacare.28.1.164. [PubMed: 15616252].

56. Heerspink HJ, Desai M, Jardine M, Balis D, Meininger G, Perkovic V. Canagliflozin slows progression of renal function decline independently of glycemic effects. J Am Soc Nephrol. 2017;28(1):368-75. doi: 10.1681/ASN.2016030278. [PubMed: 27539604]. [PubMed Central: PMC5198289]. 